\title{
Three-Phase Co-assembly: In Situ Incorporation of Nanoparticles into Tunable, Highly Ordered, Porous Silica Films
}

\section{Citation}

Vasquez, Yolanda, Mathias Kolle, Lidiya Mishchenko, Benjamin D. Hatton, and Joanna Aizenberg. 2014. Three-Phase Co-Assembly: In Situ Incorporation of Nanoparticles into Tunable, Highly Ordered, Porous Silica Films. ACS Photonics 1, no. 1: 53-60. doi:10.1021/ph400067z.

\section{Published Version}

doi:10.1021/ph400067z

\section{Permanent link}

http://nrs.harvard.edu/urn-3:HUL.InstRepos:33204051

\section{Terms of Use}

This article was downloaded from Harvard University's DASH repository, and is made available under the terms and conditions applicable to Open Access Policy Articles, as set forth at http:// nrs.harvard.edu/urn-3:HUL.InstRepos:dash.current.terms-of-use\#OAP

\section{Share Your Story}

The Harvard community has made this article openly available.

Please share how this access benefits you. Submit a story.

\section{Accessibility}




\title{
Three-Phase Co-Assembly: In-situ Incorporation of Nano- particles into Tunable, Highly-Ordered, Porous Silica Films**
}

\author{
Yolanda Vasquez, ${ }^{\dagger}$ Mathias Kolle, ${ }^{\ddagger}$ Lidiya Mishchenko, ${ }^{\ddagger}$ Benjamin D. Hatton, ${ }^{\ddagger} \S$ Joanna Aizenberg ${ }^{\ddagger} * *$ \\ †Department of Chemistry, Oklahoma State University, 107 Physical Science 1, Stillwater, OK 74074; ${ }^{\dagger}$ School of Engineer- \\ ing and Applied Sciences, Department of Chemistry and Chemical Biology, Kavli Institute for Bionano Science and Tech- \\ nology, Harvard University, 29 Oxford Street, Cambridge, MA 02138, ${ }^{\S}$ Materials Science and Engineering; University of \\ Toronto, Wallenberg Building Suite 140, Toronto, Ontario, Canada M5S 3E4
}

Supporting Information Placeholder

ABSTRACT: We present a reproducible, one-pot colloidal co-assembly approach that results in large-scale, highly-ordered porous silica films with embedded, uniformly-distributed, accessible gold nanoparticles. The unique coloration of these inverse opal films combines iridescence with plasmonic effects. The coupled optical properties are easily tunable either by changing the concentration of added nanoparticles to the solution before assembly or by localized growth of the embedded $\mathrm{Au}$ nanoparticles upon exposure to tetrachloroauric acid solution, after colloidal template removal. The presence of the selectively absorbing particles furthermore enhances the hue and saturation of the inverse opals color by suppressing incoherent diffuse scattering. The composition and optical properties of these films are demonstrated to be locally tunable using selective functionalization of the doped opals.

\section{INTRODUCTION}

Self-assembly of multi-functional 3D nanoporous structures is gaining considerable interest as a costeffective alternative to conventional top-down fabrication processes. In particular, the interconnected periodic array of pores in the inverse opal structure, synthesized from a sacrificial colloidal crystal template, has made it a viable bottom-up materials candidate for applications in photonics, ${ }^{1-4}$ tissue engineering, ${ }^{5}$ sensing, $,{ }^{6}, 7$ and catalysis. ${ }^{8,9}$ However, in order to utilize these structures as functional materials, tuning the inverse opal composition is extremely important. The incorporation of metal nanoparticles into inverse opals has recently attracted particular attention in the literature. ${ }^{8,10-21}$ The high surface area and photonic properties of inverse opals coupled with the plasmonic ${ }^{22,23}$ or catalytic ${ }^{24}$ properties of metal nanoparticles greatly expand the possible applications of these materials as catalysts, ${ }^{8}$ sensors, ${ }^{14,15}$ photonic structures, ${ }^{15-18}$ and in Surface-Enhanced Raman Spectroscopy (SERS). ${ }^{20}$

In most prior investigations, however, inverse opal structures were fabricated by multi-step processes involving assembly of colloidal crystals as sacrificial templates, then infiltrating the structure with a matrix material, such as an appropriate hydrolyzable alkoxide solgel precursor, allowing gelation to take place, and, subsequently, removing the colloidal template by dissolution or thermal decomposition. ${ }^{25-27}$ This technique yields a range of porous inverse opal structures with various chemical compositions including $\mathrm{SiO}_{2}, \mathrm{TiO}_{2}$, and $\mathrm{Al}_{2} \mathrm{O}_{3} .{ }^{4,}{ }^{28-30}$ Inverse opal matrix materials such as polymer precursors, ${ }^{31,32}$ nanoparticles, ${ }^{33,34}$ and vapor phase precursors $^{35-39}$ have also been deposited by infiltration. These types of synthetic approaches, by their very nature, not only result in fragile, cracked films, but do not allow for precise control of metal nanoparticle content or distribution, yielding non-uniformity in both composition and ensuing properties, and therefore limit their technological potential.

We have recently demonstrated that multi-layered, nanocomposite colloidal crystal films can be generated via co-assembly of polymeric colloidal spheres together with a silica sol-gel precursor solution, thus avoiding the infiltration stage of the process and the associated cracking of the structures (Figure 1a). ${ }^{40}$ Removal of the colloidal template (i.e. thermal decomposition) produces mechanically robust inverse opal films with minimal defects and large, centimeter-scale crystal domains. Herein, we develop a novel, three-phase co-assembly method by incorporating gold $\mathrm{(Au}$ ) nanoparticles in-situ into assembling centimeter-scale inverse opal films, which allows us to manipulate and control their composition. Using this new one-pot three-phase co-assembly method, we are able to reproducibly create robust, high- 


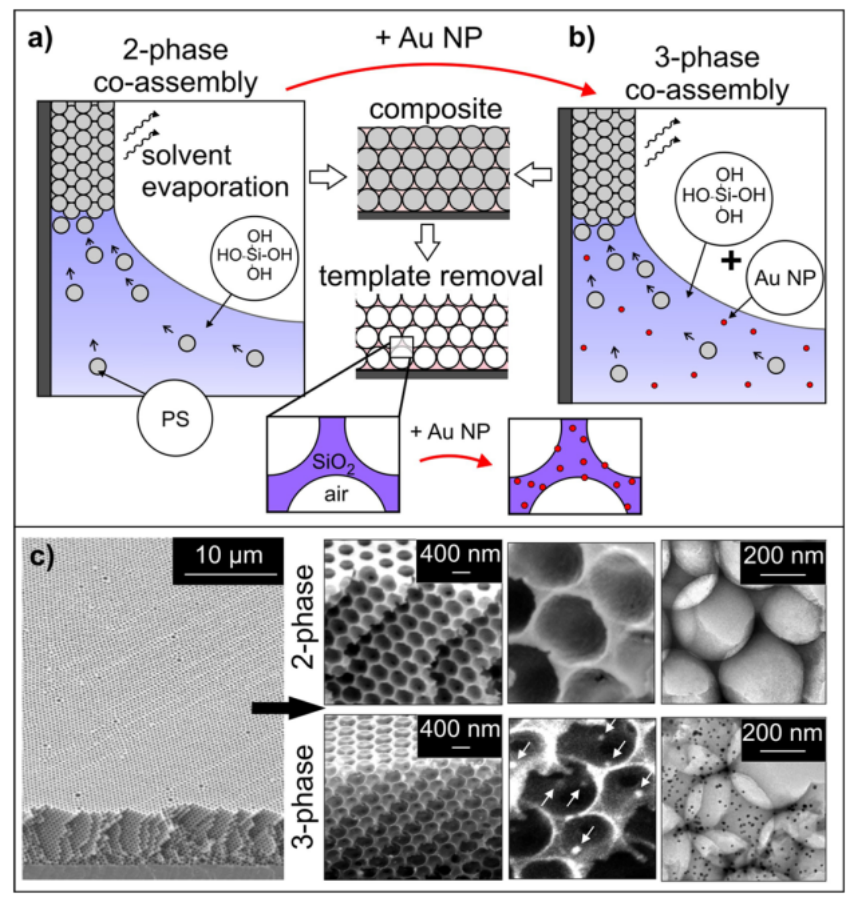

ly-ordered gold-loaded inverse opal films with uniform composition, unique optical properties, accessible functionality, and tunability for a wide range of applications.

Figure 1. Schematics of two-phase (a) and three-phase (b) coassembly methods. Both methods involved evaporative deposition of colloidal particles (340 nm, PMMA) and a hydrolyzed silica solgel precursor (TEOS) onto a vertical substrate to create a composite thin film. The organic colloidal template was then removed via calcination to create an inverse opal structure. In three-phase coassembly, gold nanoparticles assemble along with the colloids and TEOS to result in an inverse opal structure with embedded nanoparticles in the walls ("gold-loaded" opals). (c) SEM and TEM images show the large-scale, ordered, crack-free thin films that result from two- and three-phase co-assembly. SEM and TEM images of gold-loaded inverse opals (bottom) show that threephase co-assembly leads to films with uniformly distributed nanoparticles in the walls. White arrows point to the brighter contrast gold nanoparticles in the SEM image. The gold nanoparticles in the TEM image appear as dark spots.

\section{RESULTS AND DISCUSSION}

To prepare Au-containing inverse opal films, first, gold nanoparticles having a diameter of $10-15 \mathrm{~nm}$ were synthesized, ${ }^{41,42}$ isolated by centrifugation (from $15 \mathrm{~mL}$ of original solution), and added to an aqueous mixture containing $340 \mathrm{~nm}$ poly-methyl methacrylate (PMMA) colloids and a hydrolyzed silica sol-gel precursor, tetraethyl orthosilicate (TEOS) (Figure 1b). Then a glass substrate was vertically suspended in a vial containing this mixture, and a thin colloidal film was allowed to evaporatively deposit on the substrate. The resulting composite film was then calcined to remove the sacrificial PMMA template, producing a large-scale, crack-free inverse opal structure with embedded gold nanoparticles.
This one-pot synthesis of inverse opals offers a highly uniform distribution of gold nanoparticles inside the porous opal structure (Figure 1c). Note that with the use of moderate nanoparticle concentrations during

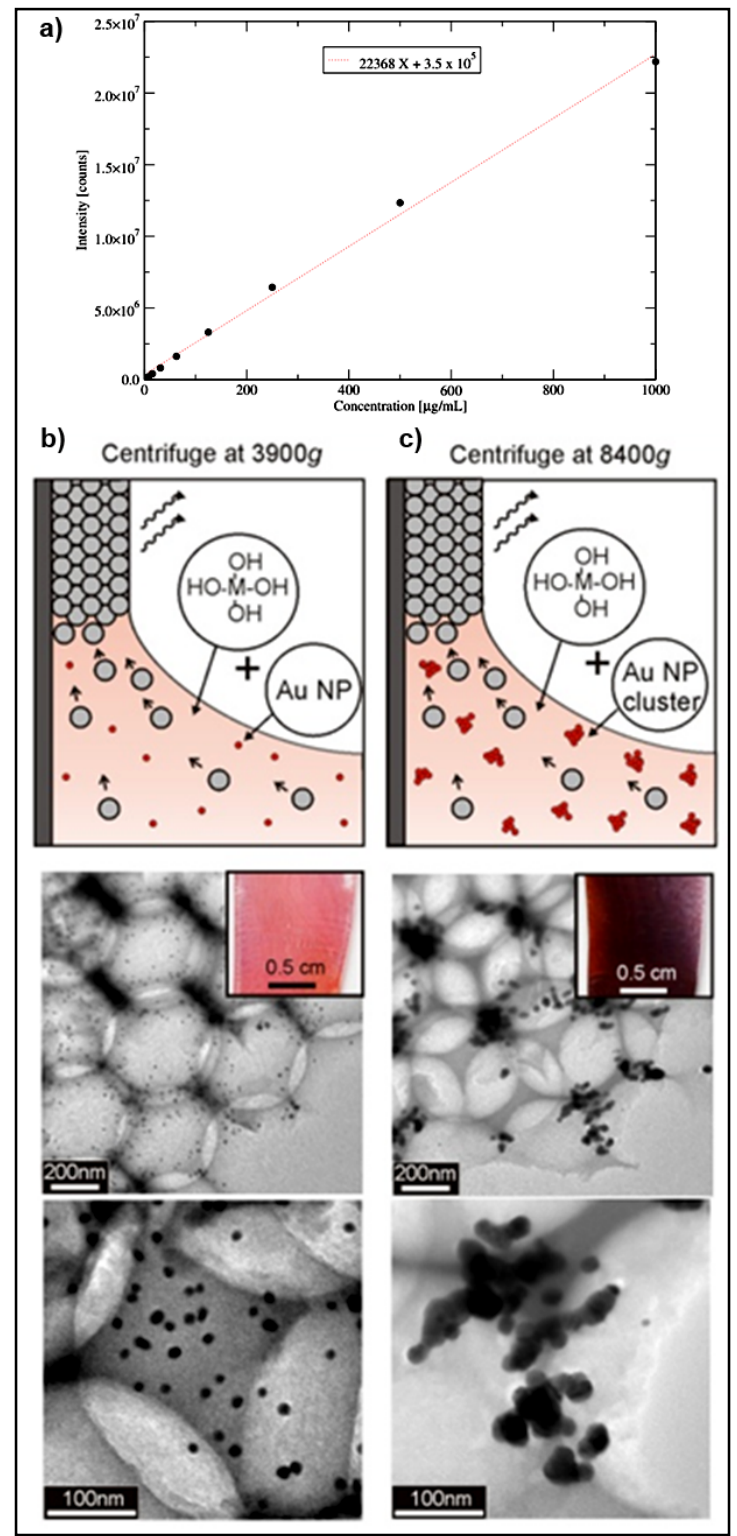

Figure 2. a) Calibration curve of Au standard generated using Inductively Coupled Plasma-Optical Emission Spectroscopy (ICPOES). Based on the standard curve and digested Au nanoparticle samples used in the inverse opals, it was determined that the [Au] $=630 \mu \mathrm{g} / \mathrm{mL}$. Taking the average size of the nanoparticles to be 10 $\mathrm{nm}$, the concentration used to make most $A u$ inverse opals was $\sim 1.2 \times 10^{-14} \mathrm{Au} \mathrm{NPs} / \mathrm{mL}$. b) Schematic and TEM images of typical Au-loaded film evaporation with nanoparticles prepared via centrifugation of the as synthesized gold solution at $3900 \mathrm{~g}$ to remove the solvent and isolate the Au NPs. The three-phase assembly results in structures with uniform Au NPs distribution. c) If the centrifugation is performed at $8400 \mathrm{~g}$, the Au NPs irreversibly cluster together (schematic) and subsequently assemble into only the larger voids of the inverse opal structure, coarsening during calcination (see TEM images below). Using a higher centrifugation speed for nanoparticle isolation results in a different gold concentration and degree of nanoparticle clustering as compared to lower centrifugation speeds, leading to altered optical properties in the films (see insets). 
assembly (approximately $6.0 \times 10^{14} \mathrm{Au} \mathrm{NPs} / \mathrm{mL}$, See Figure $2 \mathrm{a}$ ), the embedded particles in the resulting films did not have physical contact with one another. Consequently, most of the nanoparticles did not coarsen during calcination (maintaining their diameter of $10-15 \mathrm{~nm}$ ) and remained uniformly dispersed and tightly bound in the silica matrix as determined by TEM images. The uniformity of particle incorporation was also ensured by using negatively charge-stabilized gold nanoparticles in the assembling solution with similarly-charged species (colloids and silica), and low centrifugation speeds during nanoparticle isolation to prevent particle agglomeration $^{43-44}$ (Figure 2b,c).

Importantly, the highly-ordered periodic silica structure of a co-assembled inverse opal, combined with the uniform distribution of gold nanoparticles in its walls, led to interesting optical properties that reflect optical signatures of both components. The periodic silica structure is itself a photonic crystal and has an angledependent Bragg reflection peak in the visible range ( $\sim 700 \mathrm{~nm}$ at normal incidence), which lent an iridescent quality to the films. Gold nanoparticles of this size have plasmonic properties that led to selective absorption of light in the green-blue part of the spectrum $(\sim 520 \mathrm{~nm})$, lending a reddish tint to the films at all incidence angles. Optical measurements confirmed that an Au-loaded inverse opal possesses a combination of an angle-tunable reflection band of the film with an angle-independent plasmonic absorbance peak as seen in Figure 3a. The gold absorbance peak can also be easily tuned in these films by changing the concentration of gold nanoparticles added to the colloidal solution prior to film coassembly (Figure 3b), which leads to distinct differences in the color intensity depending on the amount of $\mathrm{Au}$ loaded.

Though the combination of a plasmonic peak and a Bragg reflection peak has been noted in other studies of inverse opal structures with metal nanoparticles, ${ }^{10-12,}$, 18,20 the precise control over nanoparticle concentration and uniformity in distribution is difficult, if not impossi- ble, with these previously demonstrated synthetic methods because they rely on homogeneous liquid infiltration into densely-packed colloidal crystals. However, with the three-phase co-assembly method, the uniformity in particle distribution can be easily maintained for a range of concentrations, as seen in the TEM images in Figure $3 \mathrm{c}$. The versatility of our three-phase co-assembly methodology is further demonstrated by taking advantage of the chemical accessibility of the embedded Au NPs. We show that one can tune the optical properties of the inverse opal, not only during, but also after assembly by selectively growing the NPs in the walls of the structure. This alternative tunability route has been demonstrated in the following set of experiments. Inverse opals with and without embedded gold nanoparticles were immersed in gold growth solution, containing hydroxylamine and tetrachloroauric acid $\left(\mathrm{HAuCl}_{4}\right)$, and stirred until the color of the opal began to visibly change due to nanoparticle growth (Figure 4a-c). It has been shown previously $^{45}$ that although hydroxylamine can reduce $\mathrm{Au}^{3+}$ ions (from $\mathrm{HAuCl}_{4}$ ) into bulk gold ( $\mathrm{Au}$ ), this reduction is dramatically accelerated if the solution is exposed to gold surfaces. We capitalized on this phenomenon and optimized the Au deposition conditions in our system, such that no new particle nucleation occurred in the growth solution, while almost all of the added $\mathrm{Au}$ contributed to the growth of existing nanoparticles within the opal.

Under optimized conditions, when all-silica and Auloaded inverse opals were immersed in this selective growth solution, the all-silica structures (control) remained almost unchanged, with the exception of a few randomly nucleated nanoparticles, due to a lack of nucleating sites (Figure 4d). The gold-loaded opals, on the other hand, changed color due to nanoparticle growth (Figure 4a). As seen from the SEM and TEM images, the size of the nanoparticles in the inverse opal increased (Figures $4 \mathrm{~b}$ and $4 \mathrm{c}$ ). Thus, at the very least, the nanoparticles located at the surfaces of the inverse opal walls were accessible to the aqueous growth solution. It is

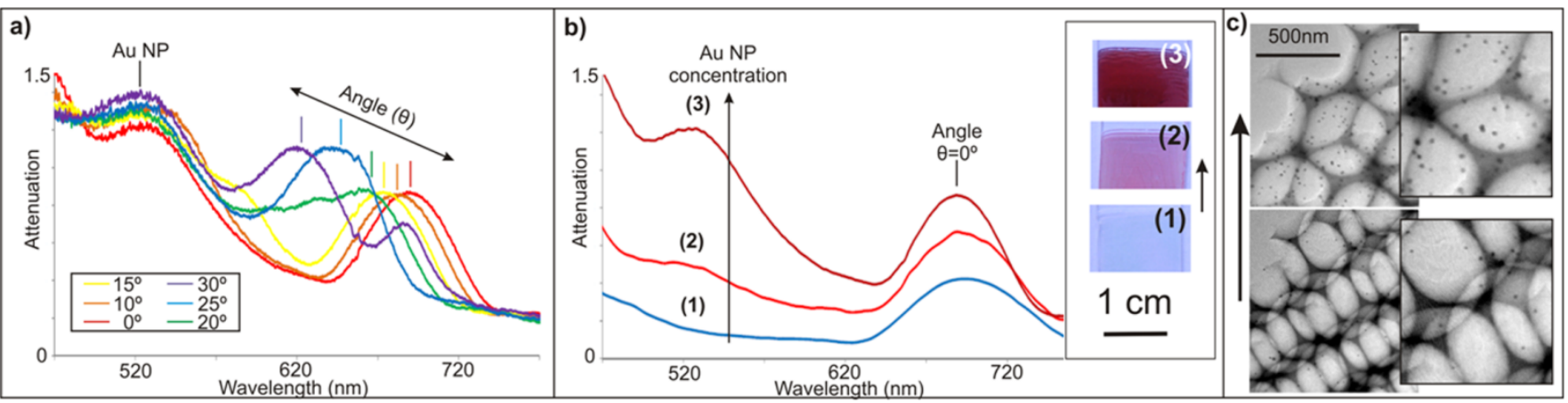

Figure 3. a) Gold-loaded inverse opals combine the angle-dependent Bragg peak of inverse opals ( $700 \mathrm{~nm}$ at normal incidence) with the angle-independent absorption peak of gold nanoparticles $(\sim 520 \mathrm{~nm})$. b) The gold absorbance peak can be tuned prior to opal assembly by adding higher amounts of gold nanoparticles to the colloidal solution. The optical images and plots correspond to three different concentrations of gold nanoparticles added to the colloidal suspension: 0 Au Np/mL (1), $1.2 \times 10^{15} \mathrm{Au} \mathrm{NPs} / \mathrm{mL}(2)$, and $7.2 \times 10^{15} \mathrm{Au} \mathrm{NPs} / \mathrm{mL}(3)$. c) TEM images correspond to inverse opals assembled with Au $6.0 \times 10^{14} \mathrm{~Np} / \mathrm{mL}$ (bottom) and $1.2 \times 10^{15} \mathrm{Au} \mathrm{NPs} / \mathrm{mL}$ (top). 


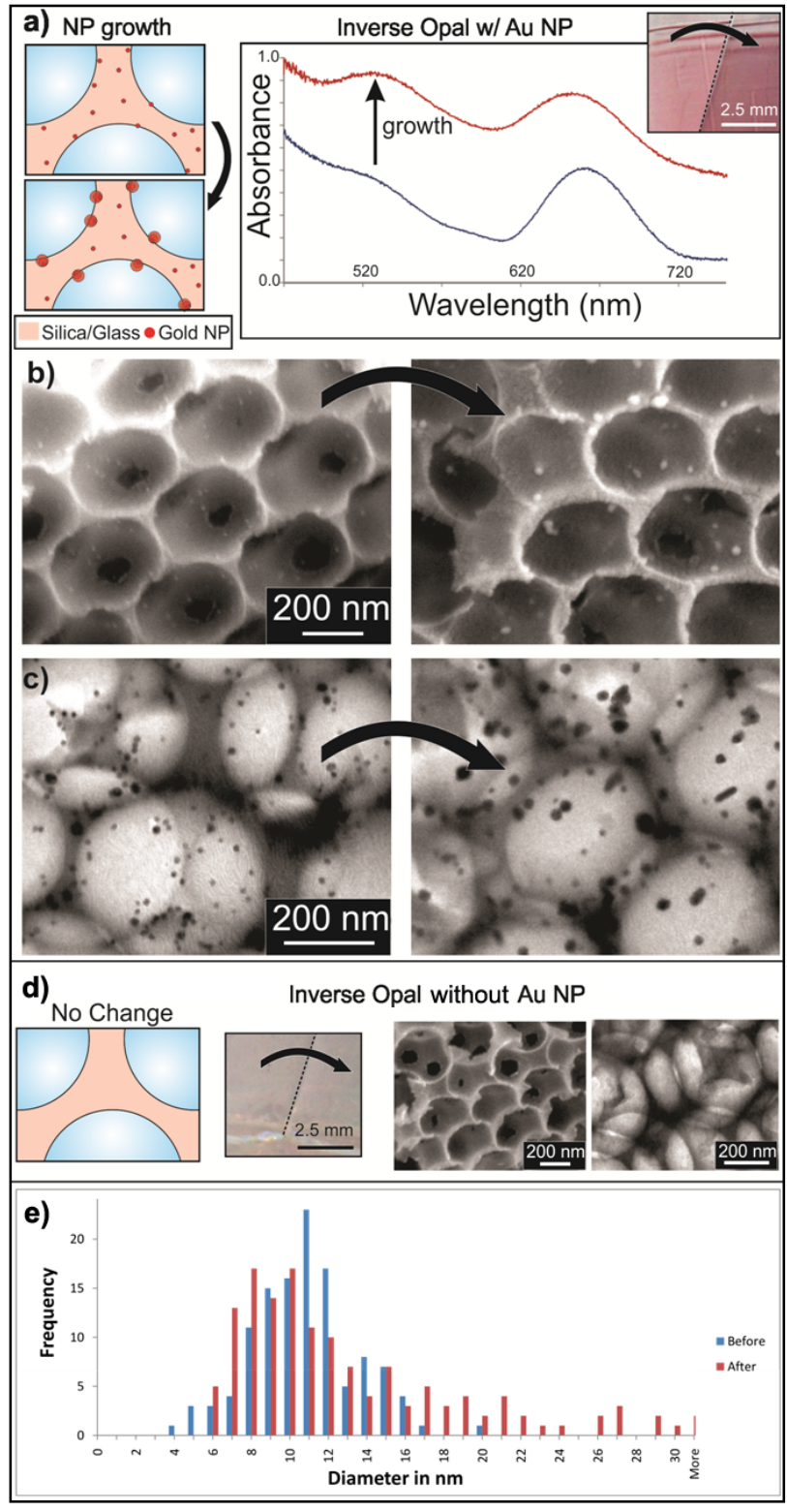

Figure 4. Inverse opals with embedded gold nanoparticles immersed in gold growth solution selectively increased the size of the preexisting gold nanoparticles. a) Gold-loaded opals changed color (optical images) due to gold nanoparticle growth, as seen in the increase of the absorption peak in the optical spectrum, b) SEM images and c) TEM images of the inverse opals with gold nanoparticles before and after growth. d) Control inverse opal structure without Au NPs shows no growth of Au NPs before and after the film was exposed to the $\mathrm{HAuCl}_{4}$ growth solution. The optical images (center) shows no change in optical properties and SEM (left) and TEM (right) images of inverse opals also confirm no growth of $\mathrm{Au}$ particles in the inverse opal. e) Diameters of Au NPs before and after exposure to the growth solution estimated using TEM images show a clear size increase and availability of embedded Au NPs.

important to note that the size effects of the larger particles may dominate the properties of these materials when the nanoparticles are not uniformly located on the surfaces and, therefore, inaccessible to growth solution. The size distribution of the particles changed, before and after re-growth, as determined by the analysis of TEM images (Figure 4e). Using a simplified "steady state" diffusion model we have also performed the modeling of the post-assembly growth of the Au nanoparticles in the inverse opal matrix and compared it to the experimental distribution observed in Figure 4e.

Our model was based on four assumptions: (i) Prior to the growth experiment, Au particles of $d=(11 \pm 3) \mathrm{nm}$ (mean \pm standard deviation, deduced from the histogram in Figure 4e) are distributed homogeneously in the solid phase of the inverse opal. (ii) The growth rate $R(x)$ depends on the distance $x$ of each particle from the inverse opal void surface, due to diffusion of precursor into the interstitial volume. (iii) $\Delta A / A=R(x)$ for the overall growth of each individual particle depends on its initial surface $A$. This means, larger particles grow faster than smaller ones. (iv) The growth rate at the surface of the inverse opal voids is constant, i.e. $R(x=0)=R_{0}$, due to an "unlimited" amount of available precursor. At a certain depth $x=x_{0}$, the growth rate vanishes, i.e. $R\left(x=x_{0}\right)=0$, due to a complete depletion of precursor by the growing particles in a shell of thickness $x=0$. This yields a linear relation of the growth rate $R$ as a function of depth $x$, given by

$$
R(x=0)=\left\{\begin{array}{cl}
R_{0}\left(1-x / x_{0}\right) & , x \leq x_{0} \\
0 & , x>x_{0}
\end{array} .\right.
$$

With these assumptions, the relevant parameters in our model are the inverse opal surface reaction rate $R_{0}$ and the depth $x_{0}$ in the interstitial matrix, at which the precursor is depleted.

The experimentally determined particle size distribution (Figure 4e) is well approximated by the model with the parameterization, $R_{0}=5$ and $x_{0}=11 \mathrm{~nm}$. While the choice for $R_{0}$ is somewhat arbitrary, the value of $x_{0}$ allows for two possible interpretations of the observed particle distribution after re-growth: (1) the precursor only penetrates to a depth comparable to the average $\mathrm{Au}$ particle diameter before being depleted, and (2) only partially exposed particles grow. The deeper the center of the particle lies within the matrix, the smaller the area exposed and the smaller the growth rate. The theoretical formulation of this problem is identical to the linear dependency found in the steady state diffusion model since the exposed area of a particle scales linearly with the depth of the particle in the matrix.

For both cases, such modeling of the particle growth based on diffusion suggests that only surface-bound particles grow as implied by the experimental results (Figure 5). Analogous template-directed growth of crystal nanoparticles from solution has been demonstrated in other systems. ${ }^{46-48}$ Interestingly, the sol-gel derived silica walls in our system are predicted to have a porosity of $\sim 2-3 \mathrm{~nm}^{49-50}$ which seems to be insufficient to allow the growth solution to access nanoparticles that are completely embedded within the inverse opal. The surfacebound growth of nanoparticles that can be controlled by the exposure of the inverse opal to the Au solution fol- 
lowing the initial assembly provides a means of finetuning the size of the particles at the surface and the associated optical properties of the films.

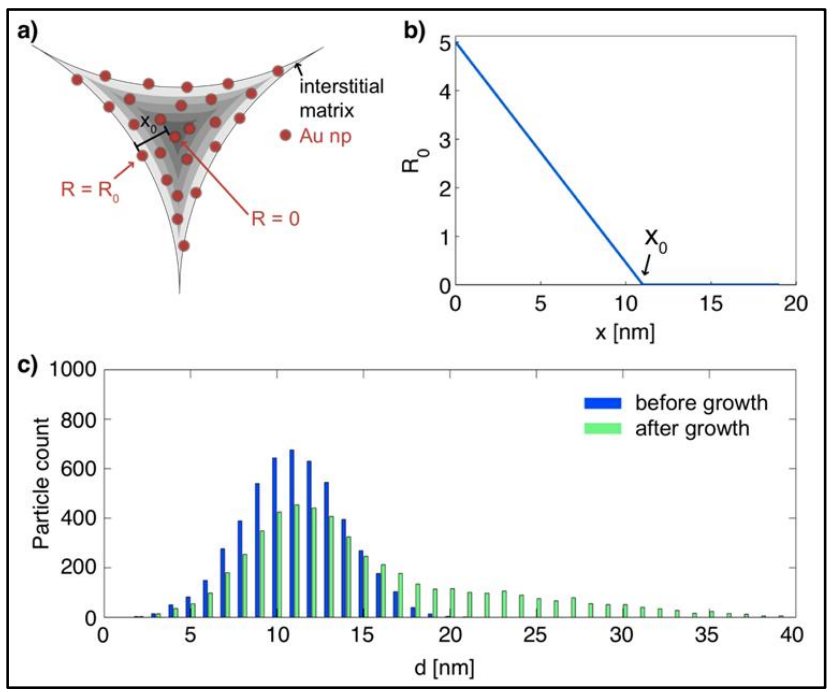

Figure 5. a) Schematic of the modeling volume with Au NPs homogeneously distributed in the inverse opal interstitial matrix. The growth rate is $R_{0}$ at the surface and 0 at a depth $x_{0}$ (gradient in gray scale in schematic). b) Growth rate as a function of depth in the interstitial volume for a steady state diffusion model. c) Calculated Au particle size distribution before (blue) and after (green) growth.

In addition to the spectral tuning achieved by tailoring the overall initial particle size during assembly, the surface-bound post-assembly growth can be exploited to modify the size-dependent spectrally selective absorption characteristics of the Au NPs at the surface only, providing further control in the engineering of the spectral composition of the inverse opal's reflection. Such surface-selective chemical accessibility of the nanoparticles significantly expands the functionality and possible applications of these large-scale inverse opals and opens a range of additional possibilities to tune the optical and catalytic properties of these films after synthesis.

Since growth of Au particles relies on the ability of the reactant solution to penetrate (i.e., wet) the inverse opal framework, we combined the aforementioned growth solution method with macro-scale chemical patterning to spatially control the wetting properties and color change in our films as another demonstration of this system's versatility. Due to the uniform, large-scale porosity and optical characteristics of co-assembled opals, we were able to use a selective functionalization technique $^{7}$ to pattern the wettability of gold-loaded inverse opals on the macroscopic scale. The opal was first rendered hydrophobic by treating it with vapor of a fluorinated silane. Then, an "M"-shaped PDMS mask was used to cover the film, and the system was exposed to oxygen plasma to render the unprotected area of the film hydrophilic (Figure 6a). Finally, the chemically patterned film was immersed in aqueous gold growth solution. Because the growth solution could only infiltrate the hydrophilic parts of the porous film (Figure 6b), a color change due to nanoparticle growth occurred only on the outside of the "M" pattern (Figure 6c). Thus, gold-loaded opals can be combined in a straightforward fashion with a selective functionalization technique to locally tune the composition and resulting properties of the inverse opal films.
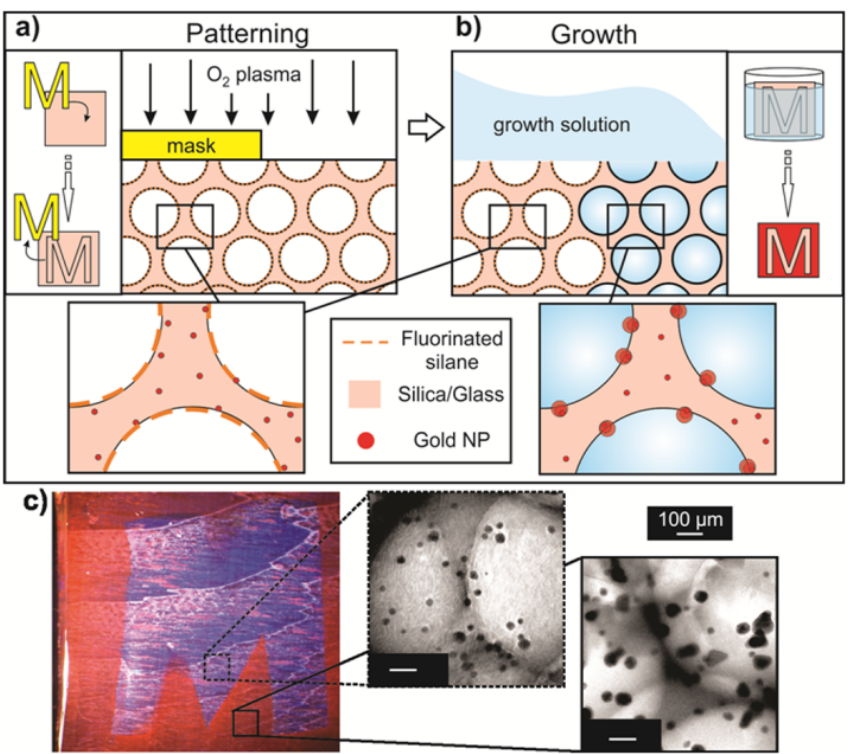

Figure 6. a) A selective functionalization technique $e^{3,4,23}$ was used to spatially pattern the wetting properties of a gold-loaded inverse opal. The opal was first rendered hydrophobic via exposure to vapor of (tridecafluoro-1,1,2,2-tetrahydrooctyl)-trichlorosilane (not shown). Part of the opal was masked with a PDMS shape ("M") and then exposed to oxygen plasma. The film remained hydrophobic in the masked region, and the rest of the film was rendered hydrophilic by oxygen plasma. b) The inverse opal with patterned wettability was then exposed to aqueous gold growth solution. The growth solution was only able to enter the hydrophilic parts of the opal, where the embedded gold nanoparticles grew in size. The hydrophobic parts of the opal remained unchanged. c) An optical image shows the patterned optical properties of an inverse opal after exposure to growth solution. TEM images (100 nm scale bars) verify that the optical contrast between the unexposed (center) and the exposed (right) regions is due to a localized growth of nanoparticles outside of the "M" shape.

\section{CONCLUSIONS}

In conclusion, we have shown that our three-phase co-assembly platform provides a versatile, one-pot approach to create highly-ordered functional inverse opal films with embedded, uniformly-distributed, accessible gold nanoparticles. The high surface area, interconnected porosity, superior compositional and structural uniformity, and accessibility of both pores and embedded nanoparticles of these films makes them a viable bottom-up materials candidate for various applications including optics and sensing. Such level of control over 
materials properties is not achievable by typical inverse opal synthesis methods. This bottom-up technique is also substantially less demanding than top-down fabrication techniques, both in terms of the equipment required and the time needed. We have shown that the coupled optical properties of the highly uniform Au-doped inverse opal films, resulting from superposition of the angle-dependent Bragg peak of inverse opals and the angle-independent absorption peak of gold nanoparticles, are easily tunable either by changing the concentration of added nanoparticles to the solution before assembly or by selectively growing the embedded nanoparticles after colloidal template removal. This is a unique aspect of our work compared to other co-assembly methods. ${ }^{21}$

The presence of the selectively absorbing particles furthermore enhances the hue and saturation of the inverse opals color by enhancing resonant scattering while suppressing incoherent diffuse scattering. ${ }^{51}$ In particular, for thin inverse opal films on reflecting surfaces (where thin film interference often results in non-negligible reflected intensity in the blue or green spectral range) this allows to create strong red hues, which are difficult to achieve without the Au NP absorption. We have also successfully demonstrated that, by using selective functionalization $^{7}$ of our doped opals, local changes in the composition and optical properties of these films can be induced. This synthetic methodology can be extended to other types of nanoparticles, such as silver, ${ }^{52}$ platinum, ${ }^{53}$, ${ }^{54}$ and palladium, which are easily synthesized and stabilized in aqueous solutions and can lend other important properties to these hybrid 3D nanoporous materials that are beneficial for antimicrobial activity, ${ }^{52} 3 \mathrm{D}$ scaffold structures for SERS sensing, ${ }^{55-56}$ colorimetric fluid analysis, ${ }^{57}$ and catalytic activity. ${ }^{24} \mathrm{We}$ believe that the robust, large-scale, nanoparticle-doped inverse opals of uniform, tunable and accessible composition synthesized via three-phase co-assembly can find use in a whole range of applications in the fields of photonics, sensing, and catalysis.

\section{EXPERIMENTAL SECTION}

Gold nanoparticle synthesis. ${ }^{42}$ All glassware used in the gold nanoparticle synthesis was thoroughly cleaned in aqua regia ( 3 parts $\mathrm{HCl}, 1$ part $\left.\mathrm{HNO}_{3}\right)($ Caution: Highly Corrosive), rinsed in triply-distilled $\mathrm{H}_{2} \mathrm{O}$, and oven-dried prior to use. The following stock solutions were prepared from triply-distilled $\mathrm{H}_{2} \mathrm{O}: 1.0 \mathrm{mM} \mathrm{HAuCl}{ }_{4}$ (Sigma Aldrich, $\mathrm{HAuCl}_{4} \cdot 3 \mathrm{H}_{2} \mathrm{O}$ salt), $38.8 \mathrm{mM}$ sodium citrate (Sigma Aldrich). In a $250 \mathrm{~mL}$ round-bottom flask equipped with a condenser, $150 \mathrm{~mL}$ of $1 \mathrm{mM} \mathrm{HAuCl}{ }_{4}$ was brought to a rolling boil with vigorous stirring ( $300 \mathrm{rpm}$, egg-shaped magnetic stir bar) in an oil bath. Rapid addition of $15 \mathrm{~mL}$ of $38.8 \mathrm{mM}$ sodium citrate to the vortex of the solution resulted in a color change from pale yellow to burgundy. Boiling was continued for $10 \mathrm{~min}$; the solution was removed from the oil bath, and stirring was continued for an additional 15 min. The solution was then allowed to reach room tempera- ture prior to use. TEM images were used to determine approximate nanoparticle size.

Film deposition. The as synthesized nanoparticle solution was centrifuged ( $3900 \mathrm{~g}$ for $60 \mathrm{~min}$, Beckman-Coulter Allegra $\mathrm{X}-22 \mathrm{R})$ in $15 \mathrm{~mL}$ batches, the supernatant was removed, and varying amounts of Au Nps $\left(6.0 \times 10^{14}-7.2 \times\right.$ $10^{15} \mathrm{Au} \mathrm{Nps} / \mathrm{mL}$ ) were re-dispersed into a $20 \mathrm{~mL}$ aqueous mixture of a $0.16 \%$ vol. monodisperse colloidal suspension of sacrificial template particles $(\sim 340 \mathrm{~nm}$ poly (methyl methacrylate) (PMMA) spheres) and $210 \mu \mathrm{L}$ of silica solgel precursor solution (prepared from the acid-catalyzed hydrolysis of tetraethyl orthosilicate (TEOS)). (Details on the preparation of substrates, sol-gel solutions, and colloidal particles are described elsewhere. ${ }^{40}$ ) Glass substrates were suspended vertically in vials containing the colloid/TEOS/Au mixture. The solvent was evaporated slowly over a period of $1-2 \mathrm{~d}$ in a $65{ }^{\circ} \mathrm{C}$ oven (Memmert UFE 500 ) on a pneumatic vibration-free table, to allow the deposition of a thin film onto the suspended substrate (growing approximately $2 \mathrm{~cm} /$ day). Thereafter, the colloidal template particles were removed (by calcination for $5 \mathrm{~h}$ at $500{ }^{\circ} \mathrm{C}$ ) to result in a porous inverse opal structure. The synthetic conditions were identical for the all-silica films, except no gold nanoparticles were added to the mixture.

The appropriate choice of the centrifugation speed is critical: if higher centrifugation speeds ( $8400 \mathrm{~g}$ for $30 \mathrm{~min}$ ) were used to remove the solvent from the original gold nanoparticle solution, the nanoparticles permanently clumped together ${ }^{43}$ assembling only into the largest voids of the inverse opal structure in localized clusters (Figure $2 \mathrm{~b}, \mathrm{c})$. These nanoparticles then coarsened during calcination. Using a higher centrifugation speed resulted in different gold concentrations and degrees of nanoparticle clustering, leading to films with different compositions and optical properties than those prepared at lower speeds.

Selective functionalization. To pattern the wettability of inverse opals on the macroscopic scale, we used a selective functionalization technique. ${ }^{7,57}$ The opal was first rendered hydrophobic with a fluorinated silane (tridecafluoro1,1,2,2-tetrahydrooctyl)- trichlorosilane (Gelest Inc.) by vapor exposure in a desiccator under vacuum overnight. An "M" shaped PDMS mask was then used to cover the film, and the system was exposed to anisotropic oxygen plasma (Diener Electric GmbH Femto-A plasma cleaner, 5 min at full power) to render the unprotected area of the film hydrophilic.

Post-assembly gold growth experiments. For goldgrowth experiments, the gold-loaded opal (either uniformly hydrophilic or patterned using selective functionalization) was suspended vertically in a vial containing $30 \mathrm{~mL}$ of 0.5 $\mathrm{mM} \mathrm{HAuCl}{ }_{4}$ (Aldrich). The solution was magnetically stirred as a $400 \mu \mathrm{L}$ of $1.8 \mathrm{mM}$ aqueous hydroxylamine $\left(\mathrm{NH}_{2} \mathrm{OH}\right)$ (Aldrich) solution was rapidly injected. The resulting "growth solution" (with the immersed opal) was stirred until the color of the opal began to visibly change due to nanoparticle growth. The control sample (all-silica opal) was subjected to the same conditions as the goldloaded opal, resulting in no significant NP nucleation or 
visible color change at the same reaction time. (Note: increasing the time of immersion resulted in the increased random nucleation of nanoparticles in the all-silica (control) inverse opal.)

Optical analysis. Absorbance/attenuation spectra were collected at various angles of incidence from sample areas of $1 \mathrm{~mm}$ in diameter using a variable-angle spectroscope. A collimated beam of white light was sent through the sample and the transmitted light was collected collinear to the light incidence direction in a solid angle of $1^{\circ}$ and coupled into a fiber before being spectrally analyzed using a Maya2000Pro spectrometer (Ocean Optics). By rotating the sample, the transmission for varying angles of light incidence was measured allowing us to calculate the attenuation (losses due to absorption and scattering) as a function of incidence angle. Attenuation is defined here as $\log _{10}\left(I_{0} / I\right)$, where $I_{0}$ is the incident light intensity, and $I$ is the transmitted light intensity.

\section{AUTHOR INFORMATION}

\section{Corresponding Author}

jaiz@seas.harvard.edu

\section{Notes}

The authors declare no competing financial interests.

\section{ACKNOWLEDGMENT}

This work was supported by the AFOSR under Award \# FA9550-09-1-0669-DOD35CAP. L.M. acknowledges fellowship support from the Department of Homeland Security (DHS). M.K. acknowledges funding from the Alexander von Humboldt-Foundation.

Key Words: colloids · nanoparticles · photonics · self-assembly · structural color

\section{REFERENCES}

(1) Davis, M. E. Ordered porous materials for emerging applications. Nature 2002, 417, 813-821.

(2) Aguirre, C. I.; Reguera, E; Stein, A. Tunable Colors in Opals and Inverse Opal Photonic Crystals. Adv. Funct. Mater. 2010 , 20, 2565-2578.

(3) Guldin, S.; Hüttner, S.; Kolle, M.; Welland, M. E.; MüllerBuschbaum P.; Friend, R. H.; Steiner, U.; Tétreault, N. DyeSensitized Solar Cell Based on a Three-Dimensional Photonic Crystal. Nano Lett. 2010, 10, 2303-2309.

(4) Zhao, X. S.; Su, F. B.; Yan, Q. F.; Guo, W. P.; Bao, X. Y.; Lv, L.; Zhou, Z. C. Templating methods for preparation of porous structures. J. Mater. Chem. 2006, 16, 637-648.

(5) Lee, J.; Shanbhag, S.; Kotov, N. A. Inverted colloidal crystals as three dimensional microenvironments for cellular cocultures. J. Mater. Chem. 2006, 16, 3558-3564.

(6) Lee, K.; Asher, S. A. Photonic crystal chemical sensors: $\mathrm{pH}$ and ionic strength. J. Am. Chem. Soc. 2000, 122, 9534-9537.

(7) Burgess, I. B.; Mishchenko, L.; Hatton, B. D.; Kolle, M.; Lončar, M.; Aizenberg, J. Encoding complex wettability patterns in chemically functionalized $3 \mathrm{D}$ photonic crystals. $J$. Am. Chem. Soc. 2011, 133, 12430-12432.
(8) Guan, G. Q.; Zapf, R.; Kolb, G.; Hessel, V.; Lowe, H.; Ye, J. H.; Zentel, R. Preferential CO oxidation over catalysts with well-defined inverse opal structures in microchannels. Int. J. Hydrogen Energ. 2008, 33, 797-801.

(9) Ren, M. M.; Ravikrishna, R.; Valsaraj, K. T. Photocatalytic degradation of gaseous organic species on photonic band-gap titania. Environ. Sci. Technol. 2006, 40, 7029-7033.

(10) Rodríguez-González, B.; Salgueiriño-Maceira, V.; GarcíaSantamaría, F.; Liz-Marzán, L. M.; Fully accessible gold nanoparticles within ordered macroporous solids. Nano Lett. 2002, 2, 471-473.

(11) Wang, D.; Li, J.; Chan, C. T.; Salgueiriño-Maceira, V.; LizMarzán, L. M.; Romanov, S.; Caruso, F.; Optical properties of nanoparticle-based metallodielectric inverse opals. Small 2005, 1, 122-130.

(12) Ding, S.; Qian, W.; Tan, Y.; Wang, Y. In-situ incorporation of gold nanoparticles of desired sizes into three-dimensional macroporous matrixes. Langmuir 2006, 22, 7105-7108.

(13) Chiappini, A.; Guddala, S.; Armellini, C.; Berneschi, S.; Cacciari, I.; Duverger-Arfuso, C.; Ferrari, M.; Righini, G. C. Fabrication and characterization of colloidal crystals infiltrated with metallic nanoparticles. Proc. SPIE 2010, 7598, 75980P.

(14) Gu, Z. Z.; Horie, R.; Kubo, S.; Yamada, Y.; Fujishima, A.; Sato, O. Fabrication of metal-coated three-dimensionally ordered macroporous film and its application as a refractive index sensor. Angew. Chem., Int. Ed. 2002, 41, 1153-1156.

(15) Tan, Y.; Qian, W.; Ding, S.; Wang, Y. Gold-nanoparticleinfiltrated polystyrene inverse opals: a three-dimensional platform for generating combined optical properties. Chem. Mater. 2006, 18, 3385-3389.

(16) Wang, J.; Ahl, S.; Li, Q.; Kreiter, M.; Neumann, T.; Burkert, K.; Knolla, W.; Jonas, U. Structural and optical characterization of 3D binary colloidal crystal and inverse opal films prepared by direct co-deposition. J. Mater. Chem. 2008, 18, 981-987.

(17) Yu, A.; Meiser, F.; Cassagneau, T.; Caruso, F. Fabrication of polymer-nanoparticle composite inverse opals by a one-step electrochemical co-deposition process. Nano Lett. 2003, 4, 177181.

(18) Wang, D.; Salgueiriño-Maceira, V.; Liz-Marzán, L. M.; Caruso, F. Gold-silica inverse opals by colloidal crystal templating. Adv. Mater. 2002, 14, 908-912.

(19) Tessier, P. M.; Velev, O. D.; Kalambur, A. T.; Lenhoff, A. M.; Rabolt, J. F.; Kaler, E. W. Structured metallic films for spectroscopic applications via colloidal crystal templating. $A d v$. Mater. 2001, 13, 396-400.

(20) Kubo, S.; Gu, Z.-Z.; Tryk, D. A.; Ohko, Y.; Sato, O.; Fujishima, A. Metal-coated colloidal crystal film as surfaceenhanced Raman scattering substrate. Langmuir 2002, 18 , 5043-5046.

(21) Cai, Z; Liu, Y. J.; Lu, X.; Teng, J. In situ "doping" inverse silica opals with size-controllable gold nanoparticles for refractive index sensing. J. Phys. Chem. C 2013, 117, 9440-9445.

(22) Kelly, K. L.; Coronado, E.; Zhao, L. L.; Schatz, G. C. The optical properties of metal nanoparticles: the influence of size, shape, and dielectric environmnent. J. Phys. Chem. B 2003, 107, 668-677.

(23) Fedlheim, D. L.; Foss, C. A. in Metal Nanoparticles: Synthesis Characterization \& Applications (Eds.: D. L. Fedlheim, C. A. Foss), Marcel Dekker, New York, 2002.

(24) Moshfegh, A. Z. Nanoparticle catalysts. J. Phys. D: Appl. Phys. 2009, 42, 233001.

(25) Wang, L.; Zhao, X. S.; Fabrication of crack-free colloidal crystals using a modified vertical deposition method. J. Phys. Chem. C 2007, 111, 8538-8542.

(26) Stein, A.; Li, F.; Denny, N. R. Morphological control in colloidal crystal templating of inverse opals, hierarchical structures and shaped particles. Chem. Mater. 2008, 20, 649666. 
(27) Velev, O. D.; Kaler, E. W. Structured porous materials via colloidal templating: from inorganic oxides to metals. $A d v$. Mater. 2000, 12, 531-534.

(28) Lytle, J. C.; Stein, A. Recent progress in syntheses and applications of inverse opals and related macroporous materials prepared by colloidal crystal templating. Ann. Rev. Nano Res. 2006, 1, 1-79.

(29) Velev, O. D.; Lenhoff, A. M. Colloidal crystals as templates for porous materials. Curr. Opin. Colloid Interface Sci. 2000, 5 , 56-63.

(30) Stein, A.; Schroden, R. C. Colloidal crystal templating of threedimensionally ordered macroporous metals prepared by colloidal crystal templating. Curr. Opin. Solid State Mater. Sci. 2001, 5, 553-564.

(31) Johnson, S. A.; Ollivier, P. J.; Mallouk, T. E. Ordered mesoporous polymers of tunable pore size from colloidal silica templates. Science 1999, 283, 963-965.

(32) Miguez, H.; Meseguer, F.; Lopez, C.; Lopez-Tejeira, F.; Sanchez-Dehesa, J. Synthesis and photonic bandgap characterization of polymer inverse opals. Adv. Mater. 2001 , 13, 393-396.

(33) Gu, Z.; Fujishima, A; Sato; O. Fabrication of high-quality opal films with controllable thickness. Chem. Mater. 2002, 14, 760765.

(34) Shimmin, R. G.; Vajtai, R.; Siegel, R. W.; Braun, P. V. Roomtemperature assembly of germanium photonic crystals through colloidal crystal templating. Chem. Mater. 2007, 19, 21022107.

(35) Blanco, A.. Chomski, E.. Grabtchak, S.. Ibisate, M.. John, S., Leonard, S. W.; Lopez, C.; Meseguer, F.; Miguez, H.; Mondia, J. P.; Ozin, G. A.; Toader, O.; van Driel, H. M. Large scale synthesis of a silicon photonic crystal with a complete threedimensional bandgap near 1.5 micrometres. Nature 2000, 405, 437-440.

(36) King, J. S.; Heineman, D.; Graungnard, E.; Summers, C. J. Atomic layer deposition in porous structures: 3D photonic crystals. Appl. Surf. Sci. 2005, 244, 511-516.

(37) Scharrer, M.; Wu, X.; Yamilov, A.; Cao, H.; Chang, R. P. H. Fabrication of inverse opal $\mathrm{ZnO}$ photonic crystals by atomic layer deposition, Appl. Phys. Lett. 2005, 86, 151113.

(38) Rugge, A.; Becker, J. S.; Gordon, R. G.; Tolbert, S. H. Tungsten nitride inverse opals by atomic layer deposition. Nano Lett. 2003, 3, 1293-1297.

(39) Rinnie, S. A.; Garcia-Santamaria, F.; Braun, P. V. Embedded cavities and waveguides in three-dimensional silicon photonic crystals. Nat. Photonics 2007, 2, 52-56.

(40) Hatton, B.; Mishchenko, L.; Davis, S.; Sandhage, K. H.; Aizenberg, J. Assembly of large-area, highly ordered, crackfree inverse opal films. Proc. Natl. Acad. Sci. U. S. A. 2010, 107, 10354-10359.

(41) McFarland, A. D.; Haynes, C. L.; Mirkin, C. A.; Van Duyne, R. P.; Godwin, H. A. Color my nanoworld. J. Chem. Educ. 2004, $81,544 \mathrm{~A}-544 \mathrm{~B}$.
(42) Grabar, K. C.; Freeman, R. G.; Hommer, M. B.; Natan, M. J. Preparation and characterization of Au colloid monolayers. Anal. Chem. 1995, 67, 735-743.

(43) Roca, M.; Pandya, N. H.; Nath, S.; Haes, A. J. Linear assembly of gold nanoparticles clusters via centrifugation. Langmuir 2010, 26, 2035-2041.

(44) Xu, J.; Perry, C. C. A novel approach to $\mathrm{Au} @ \mathrm{SiO}_{2}$ core-shell spheres. J. Non-Cryst. Solids 2007, 353, 1212-1215.

(45) Brown, K. R.; Natan, M. J. Hydroxylamine seeding of colloidal Au nanoparticles in solution on surfaces. Langmuir 1998, 14, 726-728.

(46) Klem, M. T.; Mosolf, J.; Young, M.; Douglas, T. Photochemical mineralization of europium, titanium, and ironoxyhydroxide nanoparticles in ferritin protein cage. Inorg. Chem. 2008, 47, 2237-2239;

(47) Li, M.; Schnablegger, H.; Mann, S. Coupled synthesis and selfassembly of nanoparticles to give structures with controlled organization. Nature 1999, 402, 393-395.

(48) Meldrum, F. C.; Colfen, H. Controlling mineral morphologies and structures in biological and synthetic systems. Chem. Rev. 2008, 108, 4332-4432.

(49) Brinker, C. J.; Mukherjee, S. P. Comparisons of sol-gel-derived thin films with monoliths in a multicomponent silicate glass system. Thin Solid Films 1981, 77, 141-148.

(50) Takahashi, R.; Sato, S.; Sodesawa, T.; Kawakita, M.; Ogura, K. High surface-area silica with controlled pore size prepared from nanocomposite of silica and citric acid. J. Phys. Chem. B 2000, 104, 12184-12191.

(51) Pursiainen, O. L. J.; Baumberg, J. J.; Winkler, H.; Viel, B.; Spahn, P.; Ruhl, T. Nanoparticle-tuned structural color from polymer opals. Opt. Express 2007, 15, 9553-9561.

(52) Kim, J. S.; Kuk, E.; Yu, K. N.; Kim, J. H.; Park, S. J.; Lee, H. J.; Kim, S. H.; Park, Y. K.; Park, Y. H.; Hwang, C. Y..Antimicrobial effects of silver nanoparticles. Nanomedicine: Nanotechnology, Biology and Medicine 2007, 3, 95-101.

(53) Turkevich, J.; Miner, R. S.; Babenkova, L. Further studies on the synthesis of finely divided platinum. J. Phys. Chem. 1986, 90, 4765-4767.

(54) Aika, K.; Ban, L. L.; Okura, I.; Namba, S.; Turkevich, J. Chemisorption and catalytic activity of a set of platinum catalysts. J. Res. Inst. Catal., Hokkaido Univ. 1976, 24, 54-64.

(55) Banholzer, M. J.; Millstone, J. E.; Qin, L.; Mirkin, C. A. Rationally designed nanostructures for surface-enhanced Raman scattering. Chem. Soc. Rev. 2008, 37, 885-897.

(56) He, L.; Huang, J.; Xy, T.; Chen, L.; Zhang, K.; Han, S.; He, Y.; Lee, S. T. Silver nanosheet-coated inverse opal film as a highly active and uniform SERS substrate. J. Mater. Chem. 2012, 22, 1370-1374.

(57) Raymond, K. P.; Burgess, I. B.; Kinney, M. H.; Loncar, M.; Aizenberg, J. Combinatorial wetting in colour: an optofluidic nose. Lab Chip 2012, 12, 3666-3669. 
Three-Phase Co-Assembly: In-situ Incorporation of Nanoparticles into Tunable, Highly-Ordered, Porous Silica Films

Dr. Yolanda Vasquez, Dr. Mathias Kolle, Dr. Lidiya Mishchenko, Dr. Benjamin D. Hatton, Prof. Joanna Aizenberg*

A reproducible one-pot colloidal co-assembly approach results in large-scale, highly-ordered porous silica films with embedded, uniformly-distributed, accessible gold nanoparticles. The combination of iridescence with plasmonic effects in these inverse opals gives rise to a unique coloration that can be locally tuned by post-manufacture modification of their composition and optical properties. The films' high surface area, interconnected porosity, superior compositional and structural uniformity, and accessibility of both pores and embedded nanoparticles makes them a viable self-assembly-based, bottom-up material platform for various applications in optics and sensing.

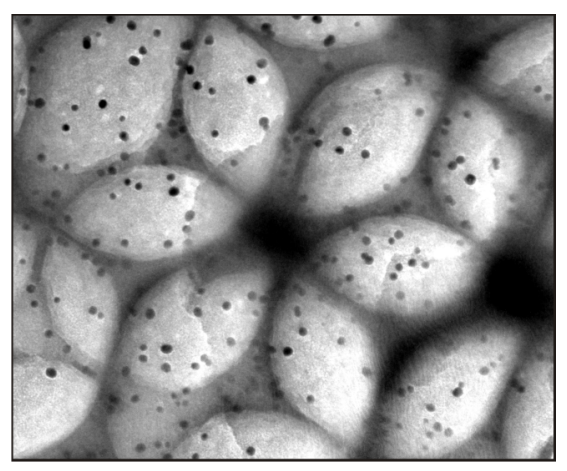

or
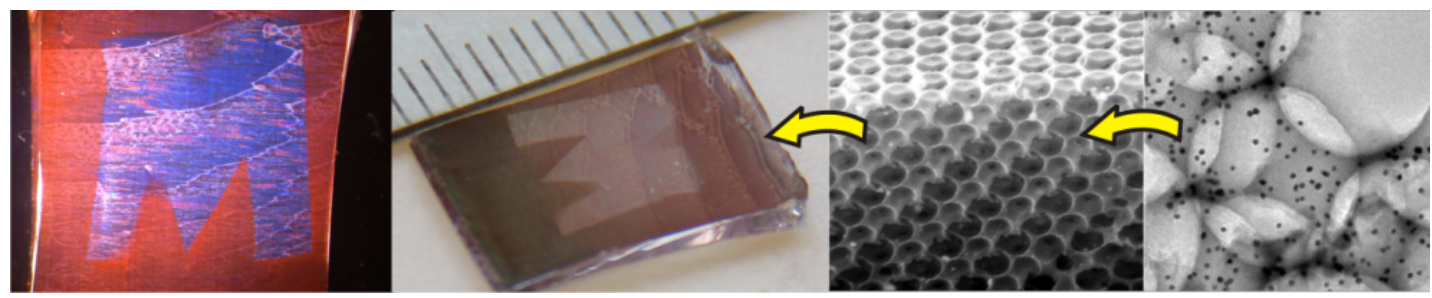